\title{
Experimental study of thermocapillary convection on a liquid bridge consisting of fluid with low Prandtl number in a floating half-zone*
}

\author{
SUN Zhiwei (孙杫伟), \\ (General Establishment of Space Science and Applications, Chinese Academy of Sciences, Beijing 100080, China) \\ HAN Jinhu（韩金虎）， \\ (Institute of Mechanics, Chinese Academy of Sciences, Beijing 100080, China) \\ DAI Lerong (戴乐蓉), \\ (Department of Chemistry, Peking University, Beijing 100871, China) \\ XIE Jingchang (解京昌) and HU Wenrui (胡文瑞) \\ (Institute of Mechanics, Chinese Academy of Sciences, Beijing 100080, China) \\ Received July 18, 1996
}

\begin{abstract}
A device of mercury liquid bridge of floating half-zone is designed to experimentally explore thermocapillary convection and its instability of a low Prandtl number liquid. Noncontacted diagnostic techniques were developed to monitor surface flow and surface deformation. The surface flow and the influence of a growing surface film (or skin) on the flow were observed. It is shown that the film is a key factor in changing the behavior associated with the thermocapillary convection. The experiment indicates that the critical Marangoni number should be much higher than that expected by the numerical simulation. The condition and process of surface film growth are discussed. The surface oscillation of the mercury bridge wrapped with "dirt-film" was observed, and the characteristics and the frequency associated with this oscillation are given.
\end{abstract}

Keywords: liquid bridge, low Prandtl nuid, microgravity, thermocapillary convection.

Marangoni convection is driven by non-uniformity of surface tension due to a temperature (or concentration) gradient along the free surface of a liquid. The liquid bridge of the floating half zone is a typical model for simulating the surface tension driven flow of crystal growth processes performed in a floating zone configuration. The steady and oscillatory convection of molten materials during the materials processing is considered one of the main causes for crystal defects and inhomogeneities. The convection driven by buoyancy is inevitable on the ground but is greatly reduced in a microgravity environment. Therefore space material science has become a new research field. In macrogravity the convection driven by surface tension gradient, or thermocapillary convection, becomes dominant. A great number of publications are devoted to the thermocapillary convection and its instability in the past two decades. However, most experimental work on this area is limited to transparent liquids with Prandtl $(\mathrm{Pr})$ number around 1 or larger ${ }^{[1-5]}$. On the other hand, from the view of applications, melts of semiconductors, metals or alloys are related to the behavior of liquids having low Prandtl numbers. These liquids are basically opaque, so the ex- 
perimental methods used for transparent media are inapplicable to these liquids. In addition, most of such media have a high melting point, large surface energy, strong absorption on the surface, and thus the surface is highly sensitive to impurities. Because of experimental difficulties, only a few on thermocapillary convection and its instability of low $\operatorname{Pr}$ liquid in literature are available ${ }^{[6-13]}$. Particularly, there has never been any report regarding the experiment on a liquid bridge in a floating half-zone of low Pr fluid. The present work is devoted to this blank area of experiment.

\section{Experiment set-up}

The liquid bridge consisting of highly purified mercury, in which the impurity-copper content is less than $10^{-6}$, was adopted as the experimental-medium, and the physical properties of mercury are listed in table 1 . Since oxidation of the mercury surface in air and absorption of other gases to the surface will result in change in the surface tension ${ }^{[14,15]}$, the system of floating halfzone is sealed in a glass tube filled with highly pure $\mathrm{N}_{2}$ ( the impurities in it are less than $0.001 \%)$. The mercury bridge is held by surface tension between two coaxial cylindrical copper (impurities $\mathrm{Fe}, \mathrm{Pb}$ and $\mathrm{Zn}$ contents in copper material are less than $10^{-6} \%$ ) rods of $d_{0}=3 \mathrm{~mm}$ in diameter. To improve wettability of the rod material to mercury, the end surfaces of the copper rods are treated with a special procedure. The upper rod is heated with temperature-controlled heater, and the lower end is cooled with ice-water during our experiment. To avoid disturbing the behavior of fluid in the liquid bridge, the temperatures at both ends are measured by copper-constantan thermocouples going through holes inside of copper rods.

Table 1 Thermo-physical parameters of mercury at $20 \mathrm{C}$

\begin{tabular}{|c|c|}
\hline Parameter & Value \\
\hline Density $\rho$ & $1.355 \times 10^{4} \mathrm{~kg}^{\prime} \mathrm{m}^{-3}$ \\
\hline Surface tension $\sigma$ & $0.4761 \mathrm{~N} \cdot \mathrm{m}^{-1}$ \\
\hline Heat capacity $C_{p}$ & $138.94 \mathrm{~J} \cdot \mathrm{kg}^{-1} \cdot \mathrm{K}^{-1}$ \\
\hline Thermal conductivity $k$ & $8.32 \mathrm{~J} \cdot \mathrm{m}^{-1} \cdot \mathrm{s}^{-1} \cdot \mathrm{K}^{-1}$ \\
\hline Thermal diffusivity $\kappa$ & $4.42 \times 10^{-6} \mathrm{~m}^{2} \cdot \mathrm{s}^{-1}$ \\
\hline Dynamical viscosity $v$ & $1.14 \times 10^{-7} \mathrm{~m}^{2} \cdot \mathrm{s}^{-1}$ \\
\hline Prandtl number Pr & 0.0258 \\
\hline Expansion coefficient $\beta$ & $0.182 \times 10^{-3} \mathrm{~K}^{-1}$ \\
\hline$\partial \sigma / \partial T$ & $0.19 \times 10^{-3} \mathrm{~N} \cdot \mathrm{m}^{-1} \cdot \mathrm{K}^{-1}$ \\
\hline
\end{tabular}

The dynamic Bond number is defined as

$$
B_{\mathrm{d}}=\rho g \beta h^{2} / \sigma_{\mathrm{T}}^{\prime}
$$

where $\rho$ is the density of the liquid, $g$, the gravitational acceleration, $h$, the height of the liquid column, $\beta$, the expansion coefficient and $\sigma_{T}^{\prime}$ the gradient of surface tension with respect to the temperature. $B_{d}$ indicates the importance of the buoyancy with respect to the gradient of surface tension. To reduce the influence of the gravity, $h$ is controlled within $3 \mathrm{~mm}\left(B_{\mathrm{d}}<1.14\right)$.

It is impossible to display an inside flow of an opaque mercury bridge with the visualization technique usually used for transparent media. We have adopted two techniques to detect a defor- 
mation or oscillation of surface of the mercury bridge. (1) A laser beam is aimed at the surface of the mercury liquid bridge. The reflected light from the curved surface is projected onto a screen. The resultant enlarged interference images on the screen are monitored by a CCD camera and recorded by a video recorder in a real time mode. Then, a variation of the interference patterns associated with the surface deformation will appear accordingly. In this way, even very small surface deformation can be identified. A typical sample of so-obtained interference pattern at one moment is shown in fig. 1. (2) The mercury surface is illuminated with a laser beam from one side. A magnifying telescope is used to monitor the reflected light from the surface and thus the enlarged light scattering pattern is recorded by a $\mathrm{CCD}$-recorder system. When surface deformation occurs, the corresponding pattern of the scattering light changes. Particularly, when the surface film exists, the pattern of the scattering light looks like a pattern of shining speckles (see fig. 2). In order to obtain the frequency of the oscillatory convection, a photocell instrument was devel-

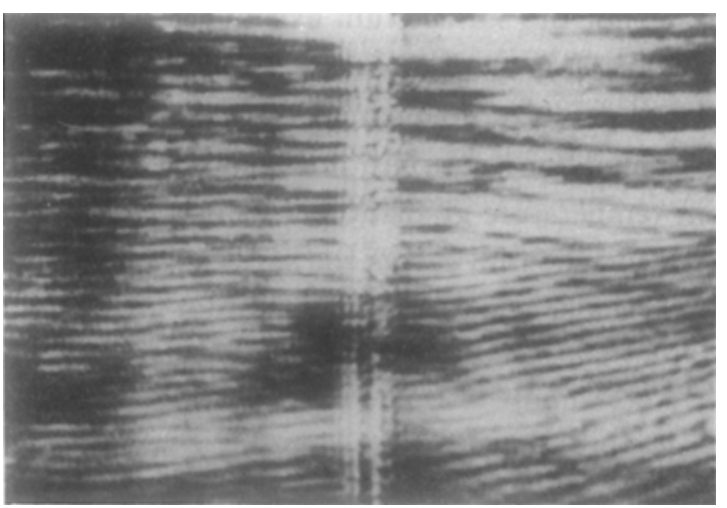

Fig. 1. The interference pattern associated with the fre' su: face configuration.

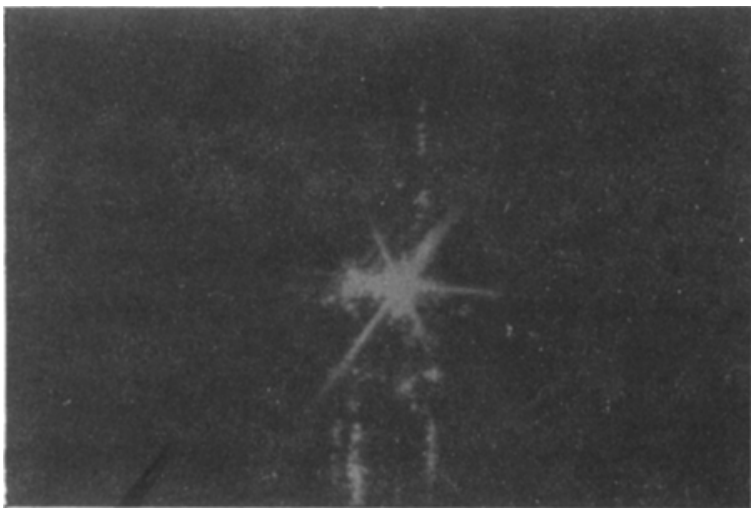

Fig. 2. The pattern of scattering speckles of the mercury surface.

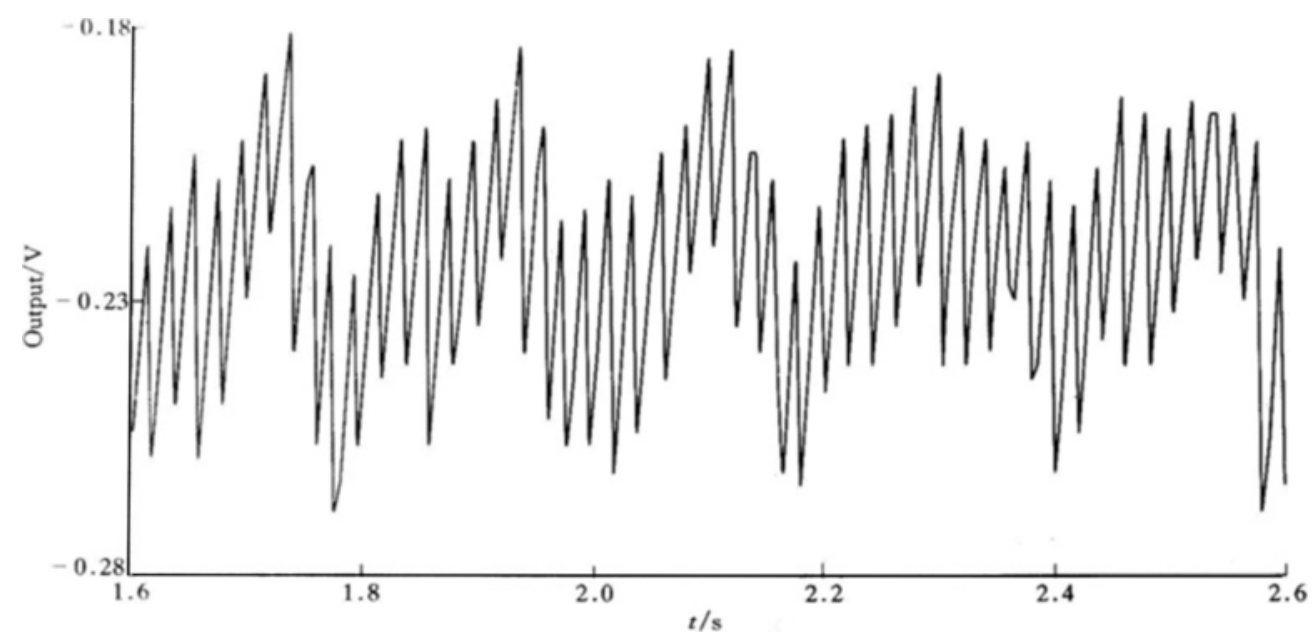

Fig. 3. The curve of photocell output associated with free surface oscillation. 
oped. The interference pattern varies after the onset of oscillation. In the present experiment, the variation of the interference pattern was recorded on a video tape, and the photocell was aimed at one of the interference fringes and fixed in place to detect the variations in the brightness resulting in a variation of output of the photocell when the fringe was moving back and forth. From the oscillation curve of the photocell output (see fig. 3 above) we can obtain the oscillation frequency.

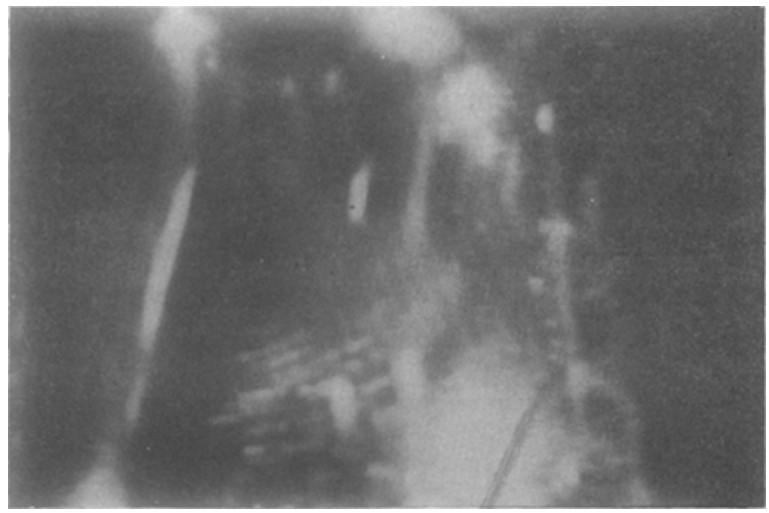

Fig. 4. The surface flow displayed by "tracers".

When a laser ray is cast on the surface of mercury, dust absorbed on the surface of the mercury (including the film growing on the surface) functions as tracers. These tracers shine brightly and shift with the liquid flow. A typical pattern of surface flow displayed by the tracers is shown in fig. 4 . Mobility of the surface is related to whether or not the surface film exists, and is therefore of great importance. When a certain temperature gradient along the surface remains but no surface flow can be observed, it implies a film suppresses the flow; under a constant temperature gradient a transition from mobility to immobility indicates the process of film growth and the film covers the surface eventually. According to this phenomenon, we can judge when and under what condition the film grows.

\section{Characteristics of the surface of mercury liquid bridge}

It seems that surface film generally exists in the liquid bridge in floating zone for low Pr liquid medium, especially when the experimental temperature is high. A discussion on the influence of growing film on surface flow of the liquid bridge is given in the present paper. When a temperature difference is applied to the liquid bridge, $1^{\circ} \mathrm{C}$ or $2^{\circ} \mathrm{C}$ is enough to stimulate the surface flow. When the hot end temperature is $12^{\circ} \mathrm{C}$, this surface mobility can last about $10 \mathrm{~min}$ and then gradually ceases. When mercury with a clean surface is used, at the beginning of the experiment the surface flow speeds up as the applied temperature difference rises, then soon slows down, and eventually ceases entirely. At first only a little shining dust can be seen, and then some speckles appear, showing flakes of surface films join the flow. These flakes multiply, growing larger and larger, and the flow slows down and finally ceases. The higher the experimental temperature is, the shorter the mobility lasts. A high temperature accelerates the formation of surface film. There are two origins of the film: mercury reacts directly with oxygen, vapour, or absorbs gases and dust; the copper rods dissolve in mercury and form a copper-mercury amalgam. To further understand the origin of the film, we used iron as the end-rod material (the solubility of iron in mercury is $1.9 \times 10^{-19} \%$ in weight, $0.03 \%$ in weight for copper) and constructed a horizontal boat. In this case, even at a high experimental temperature the mercury surface still showed a rather good degree of mobility. Thus we see that the high solubility of copper in mercury and its enrichment on mercury surface is the main cause of the surface film's formation. The solubility of copper in mercury increases quickly with the increase in temperature $\left(0.03 \%\right.$ in weight at $18^{\circ} \mathrm{C}, 0.8 \%$ in 
weight at $100^{\circ} \mathrm{C}$ ). At the beginning of an experiment, there are fewer impurities on the mercury surface; larger temperature gradient along the surface leads to a higher Marangoni flow rate. Meanwhile, higher temperature results in more impurities on mercury surface and thus makes the Marangoni flow slow down and finally suppresses the flow. A semi-quantitative analysis with an atomic emission spectroscope on the mercury in our experiment further confirms the above judgement: the copper content in used mercury is 50 times more than that before the experiment, and contents of other impurities are beyond the measurement limit of the equipment. Furthermore, when the used mercury is collected from the part close to the surface, the content of copper is 1000 times more than that before the experiment. Other impurities such as magnesium, lead (coming from rod material) also increase significantly after the experiment. This is because mercury has very high surface energy, and most of the impurities mentioned above (forming mercury amalgam) are absorbed to the mercury surface.

\section{Thermocapillary convection and its instability}

The above analysis demonstrates that maintenance of the mercury surface's mobility during the experiment is a key factor in investigating the thermocapillary convection. In this case we need to minimize the effect of the surface film. However, it is impossible to totally prevent mercury from forming amalgams. The only thing we can do is to choose lower experimental temperatures and to shorten the experimental duration. If the temperature of the hot end rod is lower than $15^{\circ} \mathrm{C}$, surface mobility can be maintained for at least five minutes. Therefore, under this condition it is possible to obtain some useful information about the thermocapillary convection and its instability ${ }^{[16]}$. A numerical simulation of liquid bridge in a floating half-zone with low Pr liquids $^{[17]}$ indicates that the critical Reynolds number corresponding to a transition from steady flow to an unsteady one is 6250 . With the parameters used in our experiment it is equivalent to about $3^{\circ} \mathrm{C}$ of a critical temperature. To further reduce the experimental temperature, dry ice was used to cool the lower end rod and the temperature of the upper end rod was kept below $15^{\circ} \mathrm{C}$. Within the 5-min duration of the experiment, the surface flow of mercury was maintained. However, there was no oscillation observed even with an applied temperature difference up to $15^{\circ} \mathrm{C}$. This fact implies that the critical temperature difference should not be lower than $15 \mathrm{C}$ which is many times higher than that estimated by the numerical simulation.

An interesting phenomenon is that a surface oscillation without surface mobility under a high applied temperature difference (in this case the lower end rod was cooled by ice-water, not dry ice) was observed. Losing surface mobility means that the mercury surface is covered with dirtfilm or skin. Therefore flow on the surface becomes impossible. With rising temperature, from the interference image and the scattering speckle pattern, we were able to see discontinuous surface deformations. We guess that these are associated with ruptures of the film. A similar phenomenon that a "dirt-film" suppressed the surface flow for transparent media with $\operatorname{Pr}=1$ was also observed experimentally by Schwabe et al.$^{[18]}$ The oscillation phenomenon which we observed has the following features: (i) the oscillation seemed to occur locally, or at least centered on some part of the surface, not as a whole like a liquid bridge of silicon oil. It looks like the oscillations come around the locations where the skin is broken during the onset of the process of oscillation. (ii) The periodicity of the oscillations seems not to be perfectly harmonic, probably because of the in- 
fluence of pieces of the skin. In comparison with large Pr liquids, the frequency of the oscillation is high, about $5 \mathrm{~Hz}$. (iii) It needs to apply higher temperature difference between the two end rods to start a transition from steady to oscillatory state than that from oscillatory to steady one. This phenomenon happens to other media as well, but it is much more outstanding when a surface film exists. The difference of these two critical temperature differences can be a few degrees ${ }^{[12]}$. In fig. 3 the oscillation process of the surface is shown, obtained by the photocell measurement for brightness variation of interference fringes, which gives the voltage output varying with time. Note that a $50 \mathrm{~Hz}$ oscillation signal, which comes from the field frequency of a TV screen, was superimposed on the signal corresponding to the surface oscillation. The experiment parameters are: $h=1.8 \mathrm{~mm}, d=3.0 \mathrm{~mm}, V / V_{0}=0.8,(\Delta T)_{\mathrm{c}}=18^{\circ} \mathrm{C}$ ( $h$ is the height of the liquid bridge; $d$ is the diameter of the end copper rods; $V_{0}$ is the volume of a cylinder with a height $h$ and diameter $d$; and $V$ is the real volume of the liquid bridge; $(\Delta T)_{c}$ is the critical temperature difference corresponding to the transition from oscillatory state to steady state). To calculate the Marangoni number we use the formula

$$
\mathrm{Ma}=\left|\sigma_{\mathrm{T}}^{\prime}\right| \Delta T h / \text { pro, }
$$

and the critical Marangoni number $(\mathrm{Ma})_{\mathrm{c}}=900$. It is interesting that this value is close to the critical Marangoni number ( 1200 ) obtained by Jurisch ${ }^{[9,10]}$. We imagine that the effect of the surface film might also have been involved in their full-zone liquid bridge experiment. On the other hand, based on direct numerical simulation for a half-zone configuration of the low Pr number fluid ${ }^{[17]}$, Levenstan et al. obtained the critical Reynolds number $(\operatorname{Re})_{c}$ to be 6250 or $(\mathrm{Ma})_{c}=$ $162(\mathrm{Ma}=\operatorname{Re} \times \operatorname{Pr}$, where $\operatorname{Re}$ is Reynolds number and $\operatorname{Pr}$ is Prandtl number). In the case of $h=$ $2 \mathrm{~mm}$, the critical temperature difference is only $2.76 \approx 3^{\circ} \mathrm{C}$.

\section{Discussion}

Jurisch et al. ${ }^{[9,10]}$ employed an electron beam equipment with a circular W-electron gun to heat the $\mathrm{Mo}$ and $\mathrm{Nb}$ rod at the center in a dynamical vacuum chamber to investigate the thermocapillary convection and its instability of floating full-zone liquid bridge. Since there is no need to apply the temperature difference by using different materials (like copper rods in our experiment), the possibility of reaction of different materials leading to surface film is eliminated and the dynamical vacuum can greatly reduce the interaction of the rod material with surrounding gases. They obtained the critical Marangoni number which is around 1200 , and the oscillation frequency which is $1 \mathrm{~Hz}$. Recently Nakamura et al. obtained the oscillation frequency of $0.5 \mathrm{~Hz}$ for their silicon material ${ }^{[13]}$. However, their experiment cannot monitor the mobility of the surface, and cannot investigate the effect of variation of applied temperature difference on thermocapillary convection either, therefore cannot determine the critical Marangoni number $(\mathrm{Ma})_{\mathrm{c}}$. In Jurisch's experiment, although the same material was used, impurities in the material may melt at high temperature and get enriched on the surface. And dynamical vacuum may confine new film growth but old skin of the material may still stay on the surface. Moreover, at high temperature, oxygen, nitrogen and hydrogen can react with $\mathrm{Nb}$. All these possible impurities may melt at high temperature and affect the features of the surface such as the temperature coefficient of the surface tension. 
The purpose of this work is to explore difficulties involved in experiment of floating half-zone liquid bridge consisting of low Pr media. Although the experimental medium is mercury, our finding may be of more general significance. For example, if the material of heating and cooling end rods is different from the material of the liquid bridge, one has to face a contradiction: a good wettability and the least mutual solubility of both materials (as metioned above, this solubility is an important reason to form the dirt-film). Jurisch et al. used the same material to bypass this problem. However the means of heating in their experiment may not be able to heat the material evenly. And more importantly, since the highest temperature is at the center of the liquid bridge, they think that in the upper half bridge convection is dominated by buoyancy and the convection of the lower part should be driven mainly by the surface tension gradient. However, it is hard to avoid mutual disturbance of the two parts. All these are potential factors which may lead to unreliable results. We believe that to monitor the mobility of the surface of the liquid bridge consisting of a low Pr medium is an important measure. Due to a high surface energy for low Pr liquid the properties of its surface may by changed by pollutants which may result in poor replicability of the experiment.

\section{Conclusion}

In this work mercury was adopted as experimental medium due to its liquid state at room temperature and its chemical stability. To explore the behavior of thermocapillary convection of a small $\mathrm{Pr}$ liquid. bridge in floating half-zone, a simple experimental set-up is designed. To detect tiny deformation of the mercury surface, two diagnostic techniques were developed which are important in finding out whether a dirt-film is on the surface. In contrast with a critical temperature $3^{2} \mathrm{C}$ predicted by numerical simulation ${ }^{[17]}$, under our experimental condition (there may be effect of the dirt-film or gases on the surface, but at least the mobility of the surface remains), it seemed that the possibility of such a small critical number for low $\operatorname{Pr}$ liquid is excluded and $(\Delta T)_{c}$ should be at least higher than $15^{\circ} \mathrm{C}$. In addition, a surface oscillation was observed for mercury liquid bridge wrapped with surface film though we could not confirm whether it really came from the thermocapillary origin.

\section{References}

1 Chang, C.E., Wilcox, W. R., Inhomogeneities due to thermocapillary flow in floating zone melting, J. Crystal Growth, $1975,28: 8$

2 Chun, C.H., Wuest, W. A microgravity simulation of the Marangoni convection, Acta Astronautica, 1978, 5:681.

3 Velten, R., Schwabe, D., Scharmann, A., Gravity-dependence of the instability of surface-tension-driven flow in floating zones, in Proceedings 7th European Symposium on Materials and Fluid Science in Microgravity (ed. Kaldeich, B. H.), ESA SP-295, Oxford, 1990, 271.

4 Cao, Z.H., Xie, J.C., Tang, Z.M. et al., The influence of buoyancy on the onset of oscillatory convection half floating zone, Advance Space Research, 1991, 11(7):163.

5 Cao, A.H., You, H.T., Tang, Z. M. et al., Experimental investigation of thermocapillary convection half floating zone, Adrance Space Research, 1991, 11(7):299.

6 Hurle, D. T. J., Jakeman, E. Effects of fluctuations on the measurement of distribution coefficients by directional solidification, J. Crystal Growth, 1969, 5:227.

7 Chang, Y.K., The float-zone growth of $\mathrm{Ti}_{3}$ Au and $\mathrm{Ti}_{3} \mathrm{Pt}, J$. Crystal Growth, 1983, 52:627.

8 Cröll, A., Müller-Sebert, W. , Nitsche, R. . Transition from steady to time-dependent Marangoni convection in partially coated silicon melt zones, in Proceedings 7 th European Symposium on Materials and Fluid Science in Microgravity, ESA SP-295, Oxford, (ed. Kaldeich, B. H. ), 1990, 263. 
9 Jurisch, M., Löser, W., Analysis of periodic non-rotational W striations in Mo single crystals due to nonsteady thermocapillary convection, J. Crystal Growth, 1990, 102:214.

10 Jurisch, M., Surface temperature oscillations of a floating zone resulting from ascillatory thermocapillary convection, $J$. Crystal Growth, 1990, 102:223.

11 Rupp, R., Auerochs, S., Müller, G. et al., Growth of GaAs single crystals by the floating zone technique under microgravity, Adv. Space Research, 1991, 11(7):297.

12 Han, J.H., Sun, Z. W., Dai, L. R. et al., Experiment on thermocapillary convection of mercury liquid bridge in floating half zone, J. Crystal Growth, 1996, 169: 129.

13 Nakamura, S., Kakimoto, K., Hibiya, T., Convection visualization and temperature fluctuation measurement in a molten silicon column, in 9 th European Symposium on Gravity Dependent Phenomena in Physical Sciences, Berlin, Germany, May 2-5, 1995 (eds. Ratke, L., Walter, H., Feuerbacher, B. ).

14 Nicholas, M.E., Joyner, P.A., Tessem, M. et al., Effect of various gases and vapours on the surface tension of mercury, J. Phys. Chem, , 1961, 65:1373.

15 Olsen, D. A., Johnson, D. C., The surface tension of mercury-thallium and mercury-indium amalgams, J. Phys. Chem., 1963,67: 2529 .

$16 \mathrm{Xie}$, J.C., Hu, W.R., Influence of heating rate on the onset of oscillation in liquid bridge of half floating zone, J. Thermal Science, 1994, 3(2): 122 .

17 Levenstam, M. , Amberg, G. . Hydrodynamical instabilities of thermocapillary flow in a half floating zone, J. Fluid Mechanics, 1995, 297:357.

18 Schwabe, D., Scharmann, A., Marangoni and buoyant convection in an open cavity under reduced and under normal gravity, Adv. Space Research, 1988, 8(12): 175 .

19 Schwabe, D. , Scharmann, A., Microgravity experiments on the transition from laminar to oscillatory thermocapillary convection in floating zones, XXV COSPAR Topical Meeting G.1, Paper G.1.2.4, Recent Scientific Results and Developments of Materials Sciences in Space. July 1984, Graz, Austria ; and also Z. Flugwiss, Weltraumforsch, 1985, 9:21. 